\title{
Willingness to Pay and Consumer's Behavioral Intentions towards Fortified Foods in Metropole
}

\author{
Satyapriya $^{1^{*}}$, Jitender Kumar Chauhan ${ }^{2}$ and Sitaram Bishnoi ${ }^{1}$ \\ ${ }^{1}$ Division of Agricultural Extension, Indian Agricultural Research Institute, \\ PUSA, New Delhi, India \\ ${ }^{2}$ Agriculture Extension, College of Post Graduate Studies in Agricultural Sciences, \\ Umiam, Meghalya (CAU, Imphal, Manipur), India \\ *Corresponding author
}

\section{A B S T R A C T}

\section{Keywords}

Behavior Intention, Fortified food, Theory of planned behavior, Willingness to pay

Article Info

\section{Accepted:}

12 December 2020

Available Online:

10 January 2021
Fortified food products are gaining popularity among consumers because of their improved awareness about their health and protection of the environment. Though, the fortified food market is lean today but to broaden it further, one must understand consumers' perception for fortified products. In the current study, willingness to pay, consumers' behavioral intention, health consciousness, level of knowledge and exploratory buying behavior for fortified was studied. To collect the data a structured questionnaire was formulated and 930 respondents residing in the urban sectors of the state of Delhi were approached. The Theory of Planned Behavior (TPB) applied to the study showed a total of 11 percentage of the variance amongst the three variables namely attitude, subjective norms and perceived behavioral control towards the behavioral intentions of the consumers about the fortified foods, and interestingly subjective norm was found to be the most impactful variable to affect the overall intention of the consumers' to purchase fortified food products. Additionally, while determining the factors for buying fortified foods, it was observed that 'nutritional composition of the product' followed by the 'food safety' and 'appropriate knowledge' about the particular product were some of the prime factors that consumers take in consideration while purchasing food products. The paper also dives into the willingness to pay estimates for the fortified food products and it was realized that consumers prefer to purchase fortified foods if they are more or less same in price value in comparison to the conventional ones. Overall, the results provided in-depth insight about the consumers' behavioral intentions, health consciousness knowledge, perception and factors contributing the purchase behavior of fortified food commodities by the Indian urban population.

\section{Introduction}

Fortified foods are foods to which extra nutrients have been added, for example the addition of nutrients including vitamin A, B, D, folic acid, iodine, and iron (Mannar, 2003). Functional foods have a wide range of applications, including medicinal, therapeutic, 
and cosmetic. According to Institute of Medicine (1994) functional foods/fortified food are defined as "any food or food ingredient that may provide a health benefit beyond the traditional nutrients it contains". Initially the original purpose of the food fortification was to eradicate the occurrence of nutritional deficiencies due to lacking of nutrients in some people's diets, or that body is unable to produce, such as targeting goiter by fortifying the edible salt with iodine etc.

It has been observed that since the decade (2005-15), there has been an overall reduction in the infant mortality rate and under-five mortality rate in India, yet the country is housing about 50 per cent of undernourished children of the world (Assocham Report-EY, 2017). Such critical situation demands for the advance food technology services in terms of fortified/functional food items that can cater to the nutritional security in the country.

Although, a rapid shift has been perceived in the diet pattern of the consumers of India, leaning more towards health food which are nutritious, safe, convenient, and affordable (Seo et al., 2014). According to the Food Safety \& Standards Regulation (2016), fortification is also recently introduced under the food regulations, which majorly aims to the mandatory fortification of the staple food/largely consumed food items of the Indian population.

As per the statistics of 2011, global sale of functional food was $€ 111$ billion, and the three largest selling markets are from the Asia pacific regions, North America and Western Europe (Bercic et al., 2016). As per the Indian context most of the functional foods and nutraceuticals are available as traditional Indian Ayurvedic Medicines. However, India's major export destination is the USA and Japan (Patwardhan et al., 2005).
Previous reviews of the scientific and commercial development of functional foods (e.g. Tapsell, 2008), has identified that expanding research into social and consumer areas is important to increase the positive influence of such health products. Also, to understand people's reasons for not using these products, research specifically should examine groups who do not currently use functional foods or supplements. Previous research has also reported that non-users of functional foods cite lack of knowledge, low perceived importance or interest in functional foods, and price as reasons to not use the products (Niva, 2006). The cognitive determinants of people's openness to consuming functional foods has been examined in previous research. A positive attitude about the importance of health, selfefficacy and people's intentions to consume foods has been found to be stronger predictor of use (Urala \& Lähteenmäki, 2004, Cox, Koster, \& Russell, 2004). In order to ascertain the reasons for increased awareness and rapid acceptance of fortified foods, combination of social, psychological, knowledge and economic based studies could throw light in view of objectives. It is also important for the consumers to recognize and characterize the fortified foods items for their better consumption, understanding, norms and motivation (Nynke et al., 2003). These factors also influence the marketing of fortified foods and such aspects indirectly reflect the overall consumer's behavior and perceptions towards purchasing the fortified food items. To date, research regarding consumer acceptability of functional foods has been limited (Paulionis, 2008).

TPB is themodel which incorporates measures of attitude and control (incorporating the notion of self-efficacy), in addition to normative perceptions, as determinants of people's behavioural decision making (Ajzen, 1991). As per TPB model, people's behaviour 
is best predicted by two variables, the intention to perform the behaviour and the individual's actual control over performing the behaviour (Armitage and Conner, 2001). Also, one individual's intentions are predicted by attitudes, the positive or negative evaluation of the performance of a behaviour, subjective norms, the perceived social pressure to perform or not perform a behaviour, and perceived behavioural control (PBC) (Ajzen, 2002).

So, it would be of great interest to study the behavioral intentions, perceptions and knowledge of the urban population towards the fortified food product consumption and the buying behavior. Such information would be vital for not only the food industry but also farmers in catering healthy products to consumers and in recognizing specific product features that could meet consumer's demand in a better way. Hence, there is a need for gaining knowledge about the consumer's behavior and consumer acceptance towards fortified foods in India. The current study is an attempt to investigate the factors affecting the buying behavior and perception of the Delhi consumers towards the fortified foods.

\section{Materials and Methods}

\section{Design and method}

The locale of the study selected was in the urban areas of Delhi NCR regions, India. Random sampling method was employed to a sample size of 930 consumer respondents. The interview schedule was designed in a custom to reveal the behavioral intentions of urban consumers for fortified food consumption. The questions were pre-tested for significance. The schedule encompasses the statements pertaining to knowledge of respondents about the fortified foods and their familiarity about the usage of the same.
Knowledge test was developed and validated that is highly responsibly for consumer behavior. Out of 26 Knowledge elements, 22 were selected based on Difficulty index ranging from 30-80\% and Discrimination index exceeding 0.20 and significant Pointbiserial correlation coefficient.

\section{Measures}

The statements for behavior intentions were divided into 7 point continuum with responses strongly agreed to strongly disagree. Demographic questions on gender and age were included at the end of the questionnaire. In order to determine the individual's values towards living their life, a value based analysis was done following the methodology given by Schwartz (1992 \& 1994). The ten value domains under the scale of 0-7 were used as a criteria to understand the respondent's credence towards lifestylerelated values. Exploratory consumer buying behavior in context of fortified food items was also measured using the exploratory buying behavior tendencies (EBBT) scale given by Baumgartner and Steenkamp (1996).

\section{TPB}

The theory of planned behavior was used to study the behavioural intention of urban consumers towards organic foods. The Theory of Planned Behavior (TPB; Ajzen, 1985) is an extension of the Theory of Reasoned Action (TRA; Ajzen \& Fishbein, 1980) which basically measures intentions to predict behaviors. Specifically, the TPB suggests that intentions are predicted by attitudes toward the behavior, subjective norms about the performance of the behavior, and extends the TRA to include perceived behavioral control (PBC; Huchting et al., 2017). Further, it is an important tool that has shown relative success in predicting food choice behaviors. In this study TPB may contribute to the structuring 
of a framework for understanding the consumer's intention to purchase fortified foods. The theory was applied to the current study and a structure model was created with the help of SPSS-AMOS (Fig. 1).

\section{Willingness}

The outcome measure, willingness, was adopted from the Pro- totype/Willingness Model (Gibbons et al., 1998). To assess openness to a trial session, a composite of two items was used to measure willingness to use the fortified products. Participants were asked to consider the following situation: "I will ONLY buy or consider buying fortified foods if they are cheaper than conventional foods. How willing would you be to try the fortified foods if they are cheaper than conventional foods". The response format was on a 7pointer scale from 1 (Strongly Agree) to 7 (Strongly Disagree).

\section{Exploratory buying behavior tendencies: EBBT}

The developed by Baumgartner and Steenkamp 1996 is a multidimensional measure using a 7-point Likert scale items ranging from 1 Strongly Disagree to 7 strongly Agree. The two components are: the exploratory acquisition of products dimension the exploratory information seeking dimension (EIS) all two components contain items specific to EBBT. In the current study the participants were asked to consider the following statement: "I would rather stick with a brand I usually buy than try something I am not very sure of', the response format was on a 7-pointer scale from 1 (Strongly Agree) to 7 (Strongly Disagree). All the data from responses of each participant were coded numerically and were analyzed statistically using the Statistical Package for Social Sciences AMOS (version 17.0).

\section{Results and Discussion}

\section{TPB}

The structural model in Fig.1 included two latent variable i.e. behavior and behavioral intention whereas, indicator variables were represented by the direct measures of Attitude (A)which basically describes an individual'slearned personality to behave in a consistent manner for the given objects, Subjective Norm (SN) that shows the supposed social pressure to perform or not to perform the task and third the Perceived Behavioral Control (PBC) which reflects the individual's self-control beliefs in taking a decision.Core parameters in the model were analyzed by Root Mean Square Error of Approximation (RMSE) and thevalue for the current model calculated was 0.110 , which falls under the category of moderate fit model. Loehlin (2004) proposed that RMSEA value (a) less than 0.08 indicates a good fit (b) 0.08 to 0.1 indicates moderate fit (c) greater than 0.1 indicates poor fit. Further it was observed that the behavioral intentions of the consumers' to purchase the fortified food items was primarily governed by the variable, subjective norm $(\mathrm{SN})$ followed by PBC $(0.05)$ and attitude (0.04). In concern with the interrelation amongst the 3 variables, data revealed that Subjective Norms had high correlation with the variable Attitude, inferring that the consumers' somewhere gets highly influenced by their significant ones while purchasing the fortified foods. Further, as per the Schwartz's value best-worst survey also, the respondents scored good ranking towards their principle to be humble with the society and to be influenced by their family, friends, relatives etc. (Fig 3).

\section{Background profile of the respondents}

It is important to understand the consumers' background in order to explore their 
understanding about the fortified food items. Results in fig 2 represents the details on the demographic profiling of the 930 respondents selected for the study. In the present study, it was observed that the 76.24 per cent of the total respondents' were females. It was reported by Bogue et al (2005) that generally females are significantly more likely to buy and consume functional food/fortified food, especially probiotic dairy products (Landstrom et al., 2007). Also, it has been noticed that females aremore positive about functional foods as compared to the males (Childs and Poryzees, 1997; Poulsen, 1999).Consequently, the higher percentage value obtained for female respondents in present study was quite expected.

Further, the relationship between fortified food consumption and age is uncertain. In the current study majority of the respondents were youth $(69.03 \%)$ i.e. in between the age group of 13- 35 years, followed by the middle age group (29.14\%) and only 1.83 percent were from the age group of above 56 years. It has been seen in the earlier studies that the vogue of consuming fortified foods is high in youths belonging to the high income groups for being healthy as compared to the old age group people (Cranfield et al., 2011; Anttolainen et al., 2001). Also, a study conducted by Markovinaet al., (2011) reported that 75 percent of the young generation (sample ofhigh school and university students) tends to buy functional foods. Whereas, quite earlier studies concluded that elderly tend to hold a more positive approach towards consuming fortified/functional food in order to place healthier food at priorities (Poulsen et al., 1999 and Steptoe et al., 1995). Hence, adults have the better understanding and subsequentlybetter comply with nutritional facts about fortified food resulting in healthier food choices (Borgmeieret al., 2009). Therefore, there is need to inform the young generation about the credibility of the fortified food and explain how they are beneficial and necessary for maintaining healthy wellbeing.

\section{Values of urban consumers}

Since, fortified foods are also known as functional food i.e. added with extra vitamins and minerals and are more nutritious, it is important to understand the underlying values of consumers particularly towards health in context of fortified food consumption. As show in fig 3, the surveyed population was found to be more concerned about their healthy as almost 60.97 percent answered it as a prime factor. Hence, the metro consumers value health the most and there is large scope for the fortified and nutritious food items. Besides the value for health, pleasing themselves followed by the importance of the significant others (friends, relatives \& family) were some of the important values for them. The least scored principle values were traditions, excitement and being prestigious.

Since, health was the prime value/driver, further insight were sought into the health consciousness of the urban consumers. A seven point continuum scale ranging from very important to not at all important was used to screen out their cognizance towards health.Table 1 reveals that the urban consumers were highly reflective and concerned about their health as the highest mean scored i.e. 5.65 was obtained for the statement indicating the concern towards health followed by the statement 2 stating health consciousness. Consequently, a specific health benefit delivered by a certain food product is anticipated to be a significant factor that affect consumers acceptance for fortified food (Siroet al., 2008). Also, it has been reported that majority of Malaysian consumers are concerned about their health consciousness and therefore had a positive 
outlook toward the consumption of green food (Salleh et al., 2010).

However, previous reports have suggested that consumers' acceptance of foods with health benefits also depends upon numerous other factors too (Frewer et al., 2003;
Verbeke, 2006; Siro et al., 2008). Limited knowledge about foods having health benefits, as well as concerns about taste and genuineness of the product are some of the identified factors responsible for multiple market failures of functional and novel foods (Onwezen and Bartels, 2011).

Table.1 Health consciousness: Perception of consumers

\begin{tabular}{|c|c|c|c|c|c|c|c|c|c|}
\hline \multirow{2}{*}{\begin{tabular}{|l|} 
Statements \\
$\begin{array}{l}\text { I am reflective and concerned } \\
\text { about my health a lot }\end{array}$ \\
\end{tabular}} & \multicolumn{2}{|c|}{ Very important } & \multicolumn{3}{|c|}{ Not at all important } & \multicolumn{2}{|c|}{ (Frequency) } & \multirow{2}{*}{$\begin{array}{l}\text { Mean } \\
5.65 \\
\end{array}$} & \multirow{2}{*}{$\begin{array}{l}\text { SD } \\
0.95\end{array}$} \\
\hline & $\begin{array}{c}181 \\
(19.46)\end{array}$ & $\begin{array}{c}299 \\
(32.15)\end{array}$ & $\begin{array}{c}392 \\
(42.15)\end{array}$ & $\begin{array}{c}28 \\
(3.01)\end{array}$ & $\begin{array}{c}27 \\
(2.90)\end{array}$ & $\begin{array}{c}1 \\
(0.10)\end{array}$ & $\begin{array}{c}2 \\
(0.21)\end{array}$ & & \\
\hline I am very health conscious & $\begin{array}{c}139 \\
(14.95)\end{array}$ & $\begin{array}{c}278 \\
(29.90)\end{array}$ & $\begin{array}{c}399 \\
(42.90)\end{array}$ & $\begin{array}{c}62 \\
(6.66)\end{array}$ & $\begin{array}{c}47 \\
(5.05)\end{array}$ & $\begin{array}{c}4 \\
(0.43)\end{array}$ & $\begin{array}{c}1 \\
(0.10)\end{array}$ & 5.41 & 1.04 \\
\hline $\begin{array}{l}\text { I usually give attention to my } \\
\text { inner feelings about my health }\end{array}$ & $\begin{array}{c}130 \\
(13.98)\end{array}$ & $\begin{array}{c}260 \\
(27.96)\end{array}$ & $\begin{array}{c}412 \\
(44.30)\end{array}$ & $\begin{array}{c}85 \\
(9.13)\end{array}$ & $\begin{array}{c}39 \\
(0.21)\end{array}$ & $\begin{array}{c}2 \\
(0.21)\end{array}$ & $\begin{array}{c}2 \\
(0.21)\end{array}$ & 5.35 & 1.01 \\
\hline $\begin{array}{l}\text { I regularly examine my health } \\
\text { status }\end{array}$ & $\begin{array}{c}87 \\
(9.35)\end{array}$ & $\begin{array}{c}192 \\
(20.65)\end{array}$ & $\begin{array}{c}386 \\
(41.50)\end{array}$ & $\begin{array}{c}127 \\
(13.65)\end{array}$ & $\begin{array}{c}125 \\
(13.44)\end{array}$ & $\begin{array}{c}5 \\
(0.53)\end{array}$ & $\begin{array}{c}8 \\
(0.86)\end{array}$ & 4.93 & 1.22 \\
\hline $\begin{array}{l}\text { I notice changes in my health } \\
\text { immediately }\end{array}$ & $\begin{array}{c}107 \\
(11.50)\end{array}$ & $\begin{array}{c}222 \\
(23.88)\end{array}$ & $\begin{array}{c}384 \\
(41.29)\end{array}$ & $\begin{array}{c}139 \\
(14.95)\end{array}$ & $\begin{array}{c}61 \\
(6.55)\end{array}$ & $\begin{array}{c}9 \\
(0.96)\end{array}$ & $\begin{array}{c}2 \\
(0.21)\end{array}$ & 5.21 & 1.93 \\
\hline Usually I am aware of my health & $\begin{array}{c}100 \\
(10.75)\end{array}$ & $\begin{array}{c}215 \\
(23.11)\end{array}$ & $\begin{array}{c}501 \\
(53.87)\end{array}$ & $\begin{array}{c}72 \\
(7.74)\end{array}$ & $\begin{array}{c}31 \\
(3.33)\end{array}$ & $\begin{array}{c}8 \\
(0.86)\end{array}$ & $\begin{array}{c}3 \\
(0.32)\end{array}$ & 5.28 & 1.01 \\
\hline $\begin{array}{l}\text { I am conscious of my state of } \\
\text { health on a daily basis }\end{array}$ & $\begin{array}{c}91 \\
(9.78)\end{array}$ & $\begin{array}{c}189 \\
(20.32)\end{array}$ & $\begin{array}{c}398 \\
(42.80)\end{array}$ & $\begin{array}{c}137 \\
(14.74)\end{array}$ & $\begin{array}{c}104 \\
(11.18)\end{array}$ & $\begin{array}{c}10 \\
(1.07)\end{array}$ & $\begin{array}{c}1 \\
(0.10)\end{array}$ & 5.00 & 1.17 \\
\hline $\begin{array}{l}\text { I notice my feelings of physical } \\
\text { state during daily hours }\end{array}$ & $\begin{array}{c}98 \\
(10.53)\end{array}$ & $\begin{array}{c}177 \\
(19.03)\end{array}$ & $\begin{array}{c}344 \\
(36.99)\end{array}$ & $\begin{array}{c}193 \\
(20.75)\end{array}$ & $\begin{array}{c}105 \\
(11.29)\end{array}$ & $\begin{array}{c}8 \\
(0.86)\end{array}$ & $\begin{array}{c}5 \\
(0.53)\end{array}$ & 4.93 & 1.22 \\
\hline $\begin{array}{l}\text { I am very involved about my } \\
\text { health issues }\end{array}$ & $\begin{array}{c}89 \\
(9.56)\end{array}$ & $\begin{array}{c}223 \\
(23.98)\end{array}$ & $\begin{array}{c}403 \\
(43.33)\end{array}$ & $\begin{array}{c}122 \\
(13.11)\end{array}$ & $\begin{array}{c}77 \\
(8.27)\end{array}$ & $\begin{array}{c}8 \\
(0.86)\end{array}$ & $\begin{array}{c}8 \\
(0.86)\end{array}$ & 5.08 & 1.16 \\
\hline
\end{tabular}

Table.2 Perception of Availability, Accessibility and affordability of fortified food

\begin{tabular}{|l|l|l|l|l|l|l|l|l|l|}
\hline Perception & \multicolumn{3}{|l}{ Extremely good } & \multicolumn{4}{|c|}{ Extremely bad } & \multicolumn{3}{|l|}{ Frequency } & Mean & SD \\
\hline $\begin{array}{l}\text { Availability of } \\
\text { fortified food }\end{array}$ & 205 & 203 & 216 & 114 & 138 & 34 & 20 & 5.04 & 1.57 \\
& $(22.04)$ & $(21.83)$ & $(23.22)$ & $(12.25)$ & $(14.84)$ & $(3.65)$ & $(3.65)$ & & \\
\hline $\begin{array}{l}\text { Accessibility of } \\
\text { Fortified food }\end{array}$ & 33 & 82 & 233 & 219 & 266 & 72 & 25 & 4.01 & 1.32 \\
\hline $\begin{array}{l}\text { Affordability of } \\
\text { fortified food }\end{array}$ & $(3.54)$ & $(8.82)$ & $(25.05)$ & $(23.54)$ & $(28.60)$ & $(7.75)$ & $(7.75)$ & & \\
\hline
\end{tabular}

Table.3 Factors important for buying decision of consumers for fortified foods 


\begin{tabular}{|c|c|c|c|c|c|c|c|c|c|}
\hline \multirow{2}{*}{$\begin{array}{l}\text { Statements } \\
\text { Lower price of fortified } \\
\text { food }\end{array}$} & \multicolumn{3}{|c|}{ Very strongly agree } & \multicolumn{2}{|c|}{ Strongly disagree } & \multicolumn{2}{|c|}{ Frequency } & \multirow{2}{*}{$\begin{array}{l}\text { Mean } \\
5.75\end{array}$} & \multirow{2}{*}{$\begin{array}{l}\text { SD } \\
0.96\end{array}$} \\
\hline & $\begin{array}{c}223 \\
(23.98)\end{array}$ & $\begin{array}{c}339 \\
(36.45)\end{array}$ & $\begin{array}{c}306 \\
(32.91)\end{array}$ & $\begin{array}{c}41 \\
(4.40) \\
\end{array}$ & $\begin{array}{c}14 \\
(1.50)\end{array}$ & $\begin{array}{c}2 \\
(0.21)\end{array}$ & $\begin{array}{c}5 \\
(0.53)\end{array}$ & & \\
\hline $\begin{array}{l}\text { More knowledge about } \\
\text { fortified food }\end{array}$ & $\begin{array}{c}220 \\
(23.66)\end{array}$ & $\begin{array}{c}379 \\
(40.75)\end{array}$ & $\begin{array}{c}307 \\
(33.01)\end{array}$ & $\begin{array}{c}13 \\
(1.39)\end{array}$ & $\begin{array}{c}8 \\
(0.86)\end{array}$ & $\begin{array}{c}2 \\
(0.21)\end{array}$ & $\begin{array}{c}1 \\
(0.10)\end{array}$ & 5.85 & 0.83 \\
\hline $\begin{array}{l}\text { Greater availability of } \\
\text { fortified food }\end{array}$ & $\begin{array}{c}183 \\
(19.68)\end{array}$ & $\begin{array}{c}377 \\
(40.53)\end{array}$ & $\begin{array}{c}323 \\
(34.73)\end{array}$ & $\begin{array}{c}40 \\
(4.30)\end{array}$ & $\begin{array}{c}5 \\
(0.53)\end{array}$ & $\begin{array}{c}1 \\
(0.10)\end{array}$ & $\begin{array}{c}1 \\
(0.10)\end{array}$ & 5.74 & 0.83 \\
\hline $\begin{array}{l}\text { More advertisement for } \\
\text { fortified food }\end{array}$ & $\begin{array}{c}197 \\
(21.18)\end{array}$ & $\begin{array}{c}317 \\
(34.08) \\
\end{array}$ & $\begin{array}{c}347 \\
(37.31)\end{array}$ & $\begin{array}{c}48 \\
(5.16) \\
\end{array}$ & $\begin{array}{c}14 \\
(1.50)\end{array}$ & $\begin{array}{c}5 \\
(0.53) \\
\end{array}$ & $\begin{array}{c}2 \\
(0.21) \\
\end{array}$ & 5.63 & 0.98 \\
\hline $\begin{array}{l}\text { Wider production of } \\
\text { biofortified crops }\end{array}$ & $\begin{array}{c}199 \\
(21.39)\end{array}$ & $\begin{array}{c}275 \\
(29.57)\end{array}$ & $\begin{array}{c}362 \\
(38.93)\end{array}$ & $\begin{array}{c}71 \\
(7.63)\end{array}$ & $\begin{array}{c}98 \\
(2.04)\end{array}$ & $\begin{array}{c}3 \\
(0.32)\end{array}$ & $\begin{array}{c}1 \\
(0.10)\end{array}$ & 4.91 & 1.22 \\
\hline $\begin{array}{l}\text { Wider product selection } \\
\text { fortified foods }\end{array}$ & $\begin{array}{c}116 \\
(12.47)\end{array}$ & $\begin{array}{c}197 \\
(21.18)\end{array}$ & $\begin{array}{c}273 \\
(29.35)\end{array}$ & $\begin{array}{c}255 \\
(27.41) \\
\end{array}$ & $\begin{array}{c}72 \\
(7.74) \\
\end{array}$ & $\begin{array}{c}14 \\
(1.50) \\
\end{array}$ & $\begin{array}{c}3 \\
(0.32) \\
\end{array}$ & 4.98 & 1.20 \\
\hline $\begin{array}{l}\text { Strong influences from } \\
\text { friends/family }\end{array}$ & $\begin{array}{c}131 \\
(14.08)\end{array}$ & $\begin{array}{c}229 \\
(24.62)\end{array}$ & $\begin{array}{c}325 \\
(34.95)\end{array}$ & $\begin{array}{c}176 \\
(18.92)\end{array}$ & $\begin{array}{c}49 \\
(5.26)\end{array}$ & $\begin{array}{c}10 \\
(1.07)\end{array}$ & $\begin{array}{c}10 \\
(1.07)\end{array}$ & 5.17 & 1.18 \\
\hline Safety & $\begin{array}{c}310 \\
(33.33)\end{array}$ & $\begin{array}{c}296 \\
(31.82)\end{array}$ & $\begin{array}{c}259 \\
(27.85)\end{array}$ & $\begin{array}{c}36 \\
(3.87)\end{array}$ & $\begin{array}{c}19 \\
(2.04)\end{array}$ & $\begin{array}{c}5 \\
(0.53)\end{array}$ & $\begin{array}{c}5 \\
(0.53)\end{array}$ & 5.90 & 1.00 \\
\hline Accepted taste & $\begin{array}{c}244 \\
(26.23)\end{array}$ & $\begin{array}{c}290 \\
(31.18)\end{array}$ & $\begin{array}{c}334 \\
(35.92)\end{array}$ & $\begin{array}{c}42 \\
(4.51)\end{array}$ & $\begin{array}{c}14 \\
(1.50)\end{array}$ & $\begin{array}{c}5 \\
(0.54)\end{array}$ & $\begin{array}{c}1 \\
(0.10)\end{array}$ & 5.75 & 0.95 \\
\hline Nutritive value & $\begin{array}{c}333 \\
(35.80) \\
\end{array}$ & $\begin{array}{c}298 \\
(32.04)\end{array}$ & $\begin{array}{c}242 \\
(26.02)\end{array}$ & $\begin{array}{c}39 \\
(4.19) \\
\end{array}$ & $\begin{array}{c}14 \\
(1.50) \\
\end{array}$ & $\begin{array}{c}2 \\
(0.21) \\
\end{array}$ & $\begin{array}{c}2 \\
(0.21) \\
\end{array}$ & 5.96 & 0.99 \\
\hline Freshness & $\begin{array}{c}282 \\
(30.32)\end{array}$ & $\begin{array}{c}263 \\
(28.27)\end{array}$ & $\begin{array}{c}247 \\
(26.55)\end{array}$ & $\begin{array}{c}114 \\
(12.25)\end{array}$ & $\begin{array}{c}23 \\
(2.47)\end{array}$ & $\begin{array}{c}1 \\
(0.10) \\
\end{array}$ & $\begin{array}{c}0 \\
(0)\end{array}$ & 5.66 & 1.12 \\
\hline Packaging & $\begin{array}{c}204 \\
(21.94)\end{array}$ & $\begin{array}{c}328 \\
(35.26)\end{array}$ & $\begin{array}{c}305 \\
(32.79)\end{array}$ & $\begin{array}{c}73 \\
(7.85) \\
\end{array}$ & $\begin{array}{c}15 \\
(1.62)\end{array}$ & $\begin{array}{c}2 \\
(0.21) \\
\end{array}$ & $\begin{array}{c}3 \\
(0.32) \\
\end{array}$ & 5.64 & 1.00 \\
\hline Brand name & $\begin{array}{c}178 \\
(19.13)\end{array}$ & $\begin{array}{c}258 \\
(27.75) \\
\end{array}$ & $\begin{array}{c}300 \\
(32.25)\end{array}$ & $\begin{array}{c}136 \\
(14.63)\end{array}$ & $\begin{array}{c}48 \\
(5.16)\end{array}$ & $\begin{array}{c}5 \\
(0.53) \\
\end{array}$ & $\begin{array}{c}5 \\
(0.53)\end{array}$ & 5.37 & 1.17 \\
\hline Simple to cook & $\begin{array}{c}218 \\
(23.44)\end{array}$ & $\begin{array}{c}270 \\
(29.03)\end{array}$ & $\begin{array}{c}331 \\
(35.60)\end{array}$ & $\begin{array}{c}95 \\
(10.21)\end{array}$ & $\begin{array}{c}8 \\
(0.86)\end{array}$ & $\begin{array}{c}6 \\
(0.64) \\
\end{array}$ & $\begin{array}{c}2 \\
(0.21) \\
\end{array}$ & 5.59 & 1.03 \\
\hline $\begin{array}{l}\text { Can be } \\
\text { tried/experimented }\end{array}$ & $\begin{array}{c}128 \\
(13.77)\end{array}$ & $\begin{array}{c}237 \\
(25.48)\end{array}$ & $\begin{array}{c}350 \\
(37.63)\end{array}$ & $\begin{array}{c}170 \\
(18.27)\end{array}$ & $\begin{array}{c}30 \\
(3.22)\end{array}$ & $\begin{array}{c}5 \\
(0.53) \\
\end{array}$ & $\begin{array}{c}10 \\
(1.07)\end{array}$ & 5.24 & 1.09 \\
\hline $\begin{array}{l}\text { Compatibility with food } \\
\text { habits }\end{array}$ & $\begin{array}{c}130 \\
(13.98)\end{array}$ & $\begin{array}{c}207 \\
(22.25)\end{array}$ & $\begin{array}{c}265 \\
(28.49)\end{array}$ & $\begin{array}{c}129 \\
(13.88)\end{array}$ & $\begin{array}{c}161 \\
(17.21)\end{array}$ & $\begin{array}{c}34 \\
(3.65)\end{array}$ & $\begin{array}{c}4 \\
(0.43)\end{array}$ & 4.87 & 1.41 \\
\hline
\end{tabular}


Table.4 WTP estimated for fortified product

\begin{tabular}{|c|c|c|c|c|c|c|c|c|c|}
\hline \multirow{2}{*}{$\begin{array}{l}\text { Statements } \\
\text { I will ONLY buy or consider buying } \\
\text { fortified foods if they are cheaper than } \\
\text { conventional foods }\end{array}$} & \multicolumn{3}{|c|}{ Very strongly agree } & \multicolumn{4}{|c|}{ Strongly disagree } & \multirow{2}{*}{$\begin{array}{l}\text { Mean } \\
4.65\end{array}$} & \multirow{2}{*}{$\begin{array}{l}\text { SD } \\
1.6\end{array}$} \\
\hline & $\begin{array}{c}116 \\
(12.47)\end{array}$ & $\begin{array}{c}117 \\
(12.60)\end{array}$ & $\begin{array}{c}285 \\
(30.65)\end{array}$ & $\begin{array}{c}171 \\
(18.38)\end{array}$ & $\begin{array}{l}225 \\
(24.19)\end{array}$ & $\begin{array}{c}7 \\
(0.75)\end{array}$ & $\begin{array}{l}9 \\
(0.96)\end{array}$ & & \\
\hline $\begin{array}{l}\text { I will ONLY buy or consider buying } \\
\text { fortified foods if they are more or less } \\
\text { the same price as conventional foods }\end{array}$ & $\begin{array}{l}69 \\
(7.41)\end{array}$ & $\begin{array}{c}159 \\
(17.09)\end{array}$ & $\begin{array}{c}424 \\
(45.60)\end{array}$ & $\begin{array}{c}157 \\
(16.89)\end{array}$ & $\begin{array}{l}103 \\
(11.07)\end{array}$ & $\begin{array}{c}10 \\
(1.07)\end{array}$ & $\begin{array}{l}8 \\
(0.86)\end{array}$ & 4.84 & 1.14 \\
\hline $\begin{array}{l}\text { I will buy or consider buying fortified } \\
\text { foods EVEN if they are slightly more } \\
\text { expensive than conventional foods }\end{array}$ & $\begin{array}{l}62 \\
(6.67)\end{array}$ & $\begin{array}{c}112 \\
(12.04)\end{array}$ & $\begin{array}{c}350 \\
(37.64)\end{array}$ & $\begin{array}{c}248 \\
(26.67)\end{array}$ & $\begin{array}{l}127 \\
(13.65)\end{array}$ & $\begin{array}{c}16 \\
(1.72)\end{array}$ & $\begin{array}{l}15 \\
(1.81)\end{array}$ & 4.59 & 1.21 \\
\hline $\begin{array}{l}\text { I will buy or consider buying fortified } \\
\text { foods EVEN if they are significantly } \\
\text { more expensive than conventional } \\
\text { foods }\end{array}$ & $\begin{array}{l}46 \\
(4.95)\end{array}$ & $\begin{array}{c}95 \\
(10.21)\end{array}$ & $\begin{array}{c}199 \\
(21.39)\end{array}$ & $\begin{array}{c}285 \\
(30.65)\end{array}$ & $\begin{array}{l}211 \\
(22.69)\end{array}$ & $\begin{array}{c}54 \\
(5.81)\end{array}$ & $\begin{array}{l}40 \\
(4.30)\end{array}$ & 4.07 & 1.37 \\
\hline
\end{tabular}

Table.5 Reasons for not consuming fortified food

\begin{tabular}{|c|c|c|c|c|c|c|c|c|c|}
\hline \multirow{2}{*}{$\begin{array}{l}\text { Statements } \\
\text { Concern about novel food }\end{array}$} & \multicolumn{2}{|c|}{ Extremely important } & \multicolumn{3}{|c|}{ Not at all important } & \multicolumn{2}{|c|}{ Frequency } & \multirow{2}{*}{$\begin{array}{l}\text { Mean } \\
5.02\end{array}$} & \multirow{2}{*}{$\begin{array}{l}\text { SD } \\
1.19\end{array}$} \\
\hline & $\begin{array}{c}119 \\
(12.80)\end{array}$ & $\begin{array}{c}195 \\
(20.97)\end{array}$ & $\begin{array}{c}296 \\
(31.83)\end{array}$ & $\begin{array}{l}235 \\
(26.34)\end{array}$ & $\begin{array}{c}56 \\
(6.02)\end{array}$ & $\begin{array}{c}16 \\
(1.72)\end{array}$ & $\begin{array}{c}3 \\
(0.32)\end{array}$ & & \\
\hline Bad taste & $\begin{array}{c}87 \\
(9.35)\end{array}$ & $\begin{array}{c}199 \\
(21.39)\end{array}$ & $\begin{array}{c}278 \\
(29.90)\end{array}$ & $\begin{array}{c}212 \\
(22.80)\end{array}$ & $\begin{array}{r}119 \\
(12.80)\end{array}$ & $\begin{array}{c}27 \\
(2.91)\end{array}$ & $\begin{array}{c}8 \\
(0.86)\end{array}$ & 4.79 & 1.29 \\
\hline I prefer non-fortified food & $\begin{array}{c}92 \\
(9.90)\end{array}$ & $\begin{array}{c}142 \\
(15.26)\end{array}$ & $\begin{array}{c}256 \\
(27.56)\end{array}$ & $\begin{array}{c}255 \\
(27.41)\end{array}$ & $\begin{array}{c}133 \\
(14.30)\end{array}$ & $\begin{array}{c}26 \\
(2.80)\end{array}$ & $\begin{array}{c}26 \\
(2.80)\end{array}$ & 4.59 & 1.38 \\
\hline $\begin{array}{l}\text { I focus more upon present } \\
\text { that future }\end{array}$ & $\begin{array}{c}97 \\
(10.43)\end{array}$ & $\begin{array}{c}170 \\
(18.27)\end{array}$ & $\begin{array}{c}286 \\
(30.75)\end{array}$ & $\begin{array}{c}217 \\
(23.33)\end{array}$ & $\begin{array}{c}122 \\
(13.11)\end{array}$ & $\begin{array}{c}28 \\
(3.01)\end{array}$ & $\begin{array}{c}11 \\
(1.07)\end{array}$ & 4.75 & 1.32 \\
\hline I am not sick & $\begin{array}{c}106 \\
(11.39)\end{array}$ & $\begin{array}{c}191 \\
(20.54)\end{array}$ & $\begin{array}{c}261 \\
(28.06)\end{array}$ & $\begin{array}{c}178 \\
(19.13)\end{array}$ & $\begin{array}{c}129 \\
(13.88)\end{array}$ & $\begin{array}{c}40 \\
(4.30) \\
\end{array}$ & $\begin{array}{c}22 \\
(2.69) \\
\end{array}$ & 4.74 & 1.45 \\
\hline Fear of side effects & $\begin{array}{c}150 \\
(16.12)\end{array}$ & $\begin{array}{c}204 \\
(21.94)\end{array}$ & $\begin{array}{c}365 \\
(39.24)\end{array}$ & $\begin{array}{c}127 \\
(13.66)\end{array}$ & $\begin{array}{c}55 \\
(5.92)\end{array}$ & $\begin{array}{c}19 \\
(2.04)\end{array}$ & $\begin{array}{c}11 \\
(1.07)\end{array}$ & 5.17 & 1.25 \\
\hline Fear of artificial additives & $\begin{array}{c}180 \\
(19.35)\end{array}$ & $\begin{array}{c}195 \\
(20.97)\end{array}$ & $\begin{array}{c}371 \\
(39.90)\end{array}$ & $\begin{array}{c}113 \\
(12.15)\end{array}$ & $\begin{array}{c}46 \\
(4.95)\end{array}$ & $\begin{array}{c}19 \\
(2.04)\end{array}$ & $\begin{array}{c}5 \\
(0.64)\end{array}$ & 5.29 & 1.22 \\
\hline Not effective & $\begin{array}{c}93 \\
(10.00)\end{array}$ & $\begin{array}{c}164 \\
(17.63)\end{array}$ & $\begin{array}{c}301 \\
(32.36)\end{array}$ & $\begin{array}{c}281 \\
30.21)\end{array}$ & $\begin{array}{c}62 \\
(6.66)\end{array}$ & $\begin{array}{c}13 \\
(1.39)\end{array}$ & $\begin{array}{c}16 \\
(1.72)\end{array}$ & 4.83 & 1.23 \\
\hline Too expensive & $\begin{array}{c}7 \\
(0.75)\end{array}$ & $\begin{array}{c}912 \\
(98.06)\end{array}$ & $\begin{array}{c}6 \\
(0.64)\end{array}$ & $\begin{array}{c}4 \\
(0.43)\end{array}$ & $\begin{array}{c}1 \\
(0.10)\end{array}$ & $\begin{array}{c}0 \\
(0)\end{array}$ & $\begin{array}{c}0 \\
(0)\end{array}$ & 5.98 & 0.25 \\
\hline Not available & $\begin{array}{c}106 \\
(11.39)\end{array}$ & $\begin{array}{c}104 \\
(11.18)\end{array}$ & $\begin{array}{c}364 \\
(39.13)\end{array}$ & $\begin{array}{c}172 \\
(18.49)\end{array}$ & $\begin{array}{c}163 \\
(17.53)\end{array}$ & $\begin{array}{c}15 \\
(1.61)\end{array}$ & $\begin{array}{c}6 \\
(0.64)\end{array}$ & 4.23 & 1.19 \\
\hline Not easy to buy & $\begin{array}{c}114 \\
(12.25)\end{array}$ & $\begin{array}{c}206 \\
(22.15)\end{array}$ & $\begin{array}{c}331 \\
(35.60)\end{array}$ & $\begin{array}{c}185 \\
(19.90)\end{array}$ & $\begin{array}{c}65 \\
(6.99)\end{array}$ & $\begin{array}{c}20 \\
(2.15)\end{array}$ & $\begin{array}{c}9 \\
(0.96)\end{array}$ & 5.02 & 1.24 \\
\hline
\end{tabular}


Table.6 Prospects for fortified food/crops

\begin{tabular}{|c|c|c|c|c|c|c|c|c|c|}
\hline \multirow{2}{*}{$\begin{array}{l}\text { Statement } \\
\text { Price of fortified foods will increase }\end{array}$} & \multicolumn{2}{|c|}{ Strongly agree } & \multicolumn{3}{|c|}{ Strongly disagreed } & \multicolumn{2}{|c|}{ Frequency } & \multirow{2}{*}{$\begin{array}{l}\text { Mean } \\
4.99\end{array}$} & \multirow{2}{*}{$\begin{array}{l}\text { SD } \\
1.55\end{array}$} \\
\hline & $\begin{array}{c}126 \\
(13.54)\end{array}$ & $\begin{array}{c}175 \\
(18.82)\end{array}$ & $\begin{array}{c}355 \\
(38.17)\end{array}$ & $\begin{array}{c}192 \\
(20.64)\end{array}$ & $\begin{array}{c}75 \\
(8.06)\end{array}$ & $\begin{array}{c}7 \\
(0.75)\end{array}$ & $\begin{array}{l}0 \\
0\end{array}$ & & \\
\hline $\begin{array}{l}\text { Even though price will rise demand } \\
\text { will also increase }\end{array}$ & $\begin{array}{c}72 \\
(7.74)\end{array}$ & $\begin{array}{c}169 \\
(18.17)\end{array}$ & $\begin{array}{c}377 \\
(40.53)\end{array}$ & $\begin{array}{c}207 \\
(22.25)\end{array}$ & $\begin{array}{c}88 \\
(9.46)\end{array}$ & $\begin{array}{c}5 \\
(0.53)\end{array}$ & $\begin{array}{c}12 \\
(1.29)\end{array}$ & 4.78 & 1.13 \\
\hline $\begin{array}{l}\text { There will be a shift from } \\
\text { conventional to fortified food } \\
\text { completely }\end{array}$ & $\begin{array}{c}73 \\
(7.85)\end{array}$ & $\begin{array}{c}160 \\
(17.20)\end{array}$ & $\begin{array}{c}258 \\
(27.75)\end{array}$ & $\begin{array}{c}252 \\
(27.09)\end{array}$ & $\begin{array}{c}161 \\
(17.31)\end{array}$ & $\begin{array}{c}12 \\
(1.29)\end{array}$ & $\begin{array}{c}14 \\
(1.50)\end{array}$ & 4.54 & 1.28 \\
\hline $\begin{array}{l}\text { Health hazards will increase due to } \\
\text { prevalent food consumption }\end{array}$ & $\begin{array}{c}105 \\
(11.29)\end{array}$ & $\begin{array}{c}167 \\
(17.96)\end{array}$ & $\begin{array}{c}295 \\
(31.73)\end{array}$ & $\begin{array}{c}256 \\
(27.52)\end{array}$ & $\begin{array}{c}83 \\
(8.93)\end{array}$ & $\begin{array}{c}16 \\
(1.72)\end{array}$ & $\begin{array}{c}8 \\
(0.86)\end{array}$ & 4.68 & 1.23 \\
\hline $\begin{array}{l}\text { There will be strict rules and } \\
\text { regulations to ensure fortified } \\
\text { certification label }\end{array}$ & $\begin{array}{c}155 \\
(16.67)\end{array}$ & $\begin{array}{c}287 \\
(30.87)\end{array}$ & $\begin{array}{c}304 \\
(32.69)\end{array}$ & $\begin{array}{c}136 \\
(14.63)\end{array}$ & $\begin{array}{c}37 \\
(3.98)\end{array}$ & $\begin{array}{c}10 \\
(1.07)\end{array}$ & $\begin{array}{c}1 \\
(0.10)\end{array}$ & 5.23 & 1.10 \\
\hline $\begin{array}{l}\text { Awareness and adoption of fortified } \\
\text { foods will increase among farmers }\end{array}$ & $\begin{array}{c}100 \\
(10.75)\end{array}$ & $\begin{array}{c}225 \\
(24.19)\end{array}$ & $\begin{array}{c}365 \\
(39.24)\end{array}$ & $\begin{array}{c}204 \\
(21.94)\end{array}$ & $\begin{array}{c}27 \\
(2.90)\end{array}$ & $\begin{array}{c}8 \\
(0.86)\end{array}$ & $\begin{array}{c}1 \\
(0.10)\end{array}$ & 5.05 & 1.04 \\
\hline $\begin{array}{l}\text { Like USA/other countries more } \\
\text { fortification will be mandatory }\end{array}$ & $\begin{array}{c}110 \\
(11.82)\end{array}$ & $\begin{array}{c}223 \\
(23.98)\end{array}$ & $\begin{array}{c}349 \\
(35.48)\end{array}$ & $\begin{array}{c}174 \\
(18.71)\end{array}$ & $\begin{array}{c}50 \\
(5.37)\end{array}$ & $\begin{array}{c}20 \\
(2.15)\end{array}$ & $\begin{array}{c}4 \\
(0.43)\end{array}$ & 4.92 & 1.16 \\
\hline $\begin{array}{l}\text { People will buy fortified food as they } \\
\text { will be more aware }\end{array}$ & $\begin{array}{c}156 \\
(16.78)\end{array}$ & $\begin{array}{c}261 \\
(28.06)\end{array}$ & $\begin{array}{c}398 \\
(42.80)\end{array}$ & $\begin{array}{c}91 \\
(9.79)\end{array}$ & $\begin{array}{c}9 \\
(0.96)\end{array}$ & $\begin{array}{c}15 \\
(1.61)\end{array}$ & $\begin{array}{l}0 \\
0\end{array}$ & 5.32 & 1.01 \\
\hline $\begin{array}{l}\text { Like in USA and Europe more } \\
\text { people will go for fortified foods }\end{array}$ & $\begin{array}{c}107 \\
(11.50)\end{array}$ & $\begin{array}{c}223 \\
(23.98)\end{array}$ & $\begin{array}{c}330 \\
(35.48)\end{array}$ & $\begin{array}{c}229 \\
(24.63)\end{array}$ & $\begin{array}{c}30 \\
(3.22)\end{array}$ & $\begin{array}{c}7 \\
(0.76)\end{array}$ & $\begin{array}{c}4 \\
(0.43)\end{array}$ & 5.02 & 2.30 \\
\hline $\begin{array}{l}\text { Fortified food market is a growing } \\
\text { market }\end{array}$ & $\begin{array}{c}134 \\
(14.40)\end{array}$ & $\begin{array}{c}230 \\
(24.74)\end{array}$ & $\begin{array}{c}449 \\
(48.27)\end{array}$ & $\begin{array}{c}94 \\
(10.10)\end{array}$ & $\begin{array}{c}12 \\
(1.29)\end{array}$ & $\begin{array}{c}7 \\
(0.76)\end{array}$ & $\begin{array}{c}4 \\
(0.43)\end{array}$ & 5.24 & 0.94 \\
\hline $\begin{array}{l}\text { Fortified food consumption will lead } \\
\text { better health }\end{array}$ & $\begin{array}{c}185 \\
(19.90)\end{array}$ & $\begin{array}{c}255 \\
(27.41)\end{array}$ & $\begin{array}{c}362 \\
(38.93)\end{array}$ & $\begin{array}{c}108 \\
(11.61)\end{array}$ & $\begin{array}{c}11 \\
(1.18)\end{array}$ & $\begin{array}{c}9 \\
(0.96)\end{array}$ & $\begin{array}{c}0 \\
(0)\end{array}$ & 5.39 & 1.03 \\
\hline $\begin{array}{l}\text { Future generation is going to be } \\
\text { more health conscious and hence } \\
\text { fortified food demand will grow }\end{array}$ & $\begin{array}{c}242 \\
(26.02)\end{array}$ & $\begin{array}{c}255 \\
(27.41)\end{array}$ & $\begin{array}{c}293 \\
(31.50)\end{array}$ & $\begin{array}{c}117 \\
(12.59)\end{array}$ & $\begin{array}{c}12 \\
(1.29)\end{array}$ & $\begin{array}{c}11 \\
(1.18)\end{array}$ & $\begin{array}{c}0 \\
(0)\end{array}$ & 5.49 & 1.11 \\
\hline
\end{tabular}

Table.7 Exploratory buying behavior tendencies (EBBT) of the consumers

\begin{tabular}{|c|c|c|c|c|c|c|c|}
\hline Statements & Strongly & gree & isagree & & equency & Mean & SD \\
\hline $\begin{array}{l}\text { Even though certain food products are available in a } \\
\text { number of different flavor. I tend to buy the same flavor }\end{array}$ & $\begin{array}{c}139 \\
(14.95)\end{array}$ & $\begin{array}{c}373 \\
(40.10)\end{array}$ & $\begin{array}{c}215 \\
(23.11)\end{array}$ & $\begin{array}{c}116 \\
(12.47)\end{array}$ & $\begin{array}{c}87 \\
(9.35)\end{array}$ & 2.52 & 1.16 \\
\hline $\begin{array}{l}\text { I would rather stick with a brand I usually buy than try } \\
\text { something I am not very sure of }\end{array}$ & $\begin{array}{c}110 \\
(11.83)\end{array}$ & $\begin{array}{c}390 \\
(41.94)\end{array}$ & $\begin{array}{c}243 \\
(26.12)\end{array}$ & $\begin{array}{c}81 \\
(8.71)\end{array}$ & $\begin{array}{c}106 \\
(11.39)\end{array}$ & 2.65 & 1.14 \\
\hline I think of myself as a brand-loyal consumer & $\begin{array}{c}102 \\
(10.97)\end{array}$ & $\begin{array}{c}314 \\
(33.77)\end{array}$ & $\begin{array}{c}319 \\
(34.30)\end{array}$ & $\begin{array}{c}102 \\
(10.96)\end{array}$ & $\begin{array}{c}93 \\
(10.00)\end{array}$ & 2.75 & 1.10 \\
\hline $\begin{array}{l}\text { When I see a new brand on the shelf, I'm not afraid of } \\
\text { giving it a try }\end{array}$ & $\begin{array}{c}87 \\
(9.35)\end{array}$ & $\begin{array}{c}419 \\
(45.05)\end{array}$ & $\begin{array}{c}305 \\
(32.80)\end{array}$ & $\begin{array}{c}49 \\
(5.26)\end{array}$ & $\begin{array}{c}70 \\
(7.52)\end{array}$ & 3.43 & 0.99 \\
\hline $\begin{array}{l}\text { When I go to a restaurant, I feel it is safer to order dishes } \\
\text { I am familiar with }\end{array}$ & $\begin{array}{c}129 \\
(13.88)\end{array}$ & $\begin{array}{c}420 \\
(45.16)\end{array}$ & $\begin{array}{c}213 \\
(22.91)\end{array}$ & $\begin{array}{c}73 \\
(7.85)\end{array}$ & $\begin{array}{c}95 \\
(10.21)\end{array}$ & 2.55 & 1.13 \\
\hline $\begin{array}{l}\text { If I like a brand, I rarely switch from it just to try } \\
\text { something different }\end{array}$ & $\begin{array}{c}79 \\
(8.49)\end{array}$ & $\begin{array}{c}412 \\
(44.30)\end{array}$ & $\begin{array}{c}238 \\
(25.60)\end{array}$ & $\begin{array}{c}86 \\
(9.24)\end{array}$ & $\begin{array}{c}115 \\
(12.36)\end{array}$ & 2.72 & 1.13 \\
\hline I am very cautious in trying new or different products & $\begin{array}{c}97 \\
(10.43)\end{array}$ & $\begin{array}{c}408 \\
(43.87)\end{array}$ & $\begin{array}{c}240 \\
(25.81)\end{array}$ & $\begin{array}{c}92 \\
(9.90)\end{array}$ & $\begin{array}{c}99 \\
(10.00)\end{array}$ & 2.67 & 1.12 \\
\hline $\begin{array}{l}\text { I enjoy taking chances in buying unfamiliar brands just } \\
\text { get some variety in my purchases }\end{array}$ & $\begin{array}{c}83 \\
(8.92)\end{array}$ & $\begin{array}{c}317 \\
(34.08)\end{array}$ & $\begin{array}{c}354 \\
(38.06)\end{array}$ & $\begin{array}{c}77 \\
(8.27)\end{array}$ & $\begin{array}{c}99 \\
(10.64)\end{array}$ & 3.22 & 1.07 \\
\hline $\begin{array}{l}\text { I rarely buy brands about which I am uncertain how } \\
\text { they will perform }\end{array}$ & $\begin{array}{c}210 \\
(22.59)\end{array}$ & $\begin{array}{c}95 \\
(10.21)\end{array}$ & $\begin{array}{c}286 \\
(30.75)\end{array}$ & $\begin{array}{c}159 \\
(17.09)\end{array}$ & $\begin{array}{c}180 \\
(19.35)\end{array}$ & 3.42 & 1.07 \\
\hline I usually eat the same kinds of foods on a regular basis & 114 & 353 & 204 & 110 & 149 & 2.81 & 1.26 \\
\hline
\end{tabular}




\begin{tabular}{|c|c|c|c|c|c|c|c|}
\hline & $(12.25)$ & $(37.96)$ & (21.94) & $(11.82)$ & $(16.02)$ & & \\
\hline $\begin{array}{l}\text { Reading mail advertising to find out what's new is a } \\
\text { waste of time }\end{array}$ & $\begin{array}{c}86 \\
(9.24)\end{array}$ & $\begin{array}{c}242 \\
(26.02)\end{array}$ & $\begin{array}{c}288 \\
(30.97)\end{array}$ & $\begin{array}{c}134 \\
(14.40)\end{array}$ & $\begin{array}{c}180 \\
(19.35)\end{array}$ & 3.08 & 1.24 \\
\hline $\begin{array}{l}\text { I like to go window shopping and find out about the } \\
\text { latest styles }\end{array}$ & $\begin{array}{c}101 \\
(10.86)\end{array}$ & $\begin{array}{c}415 \\
(44.62)\end{array}$ & $\begin{array}{c}256 \\
(27.52)\end{array}$ & $\begin{array}{c}74 \\
(7.96)\end{array}$ & $\begin{array}{c}84 \\
(9.03)\end{array}$ & 3.40 & 1.07 \\
\hline I get very bored listening to others about their purchases & $\begin{array}{c}100 \\
(10.76)\end{array}$ & $\begin{array}{c}285 \\
(30.64)\end{array}$ & $\begin{array}{c}310 \\
(33.33)\end{array}$ & $\begin{array}{c}93 \\
(10.00)\end{array}$ & $\begin{array}{c}142 \\
(15.26)\end{array}$ & 2.88 & 1.19 \\
\hline $\begin{array}{l}\text { I generally read even my junk mail just to know what it } \\
\text { is about }\end{array}$ & $\begin{array}{c}98 \\
(10.53)\end{array}$ & $\begin{array}{c}286 \\
(30.75)\end{array}$ & $\begin{array}{c}262 \\
(28.17)\end{array}$ & $\begin{array}{c}131 \\
(14.08)\end{array}$ & $\begin{array}{c}153 \\
(16.45)\end{array}$ & 3.04 & 1.23 \\
\hline I don't like to shop around just out of curiosity & $\begin{array}{c}97 \\
(10.43)\end{array}$ & $\begin{array}{c}328 \\
(35.26)\end{array}$ & $\begin{array}{c}267 \\
(28.71)\end{array}$ & $\begin{array}{c}103 \\
(11.07)\end{array}$ & $\begin{array}{c}135 \\
(14.51)\end{array}$ & 2.83 & 1.19 \\
\hline $\begin{array}{l}\text { I like to browse through mail older catalogues even when } \\
\text { I don't plan to buy anything }\end{array}$ & $\begin{array}{c}93 \\
(10.00)\end{array}$ & $\begin{array}{c}303 \\
(32.59)\end{array}$ & $\begin{array}{c}254 \\
(27.31)\end{array}$ & $\begin{array}{c}115 \\
(12.36)\end{array}$ & $\begin{array}{c}165 \\
(17.75)\end{array}$ & 3.04 & 1.24 \\
\hline $\begin{array}{l}\text { I usually throw away mail advertisement without } \\
\text { reading them }\end{array}$ & $\begin{array}{c}94 \\
(10.10)\end{array}$ & $\begin{array}{c}263 \\
(28.27)\end{array}$ & $\begin{array}{c}272 \\
(29.24)\end{array}$ & $\begin{array}{c}110 \\
(11.83)\end{array}$ & $\begin{array}{c}191 \\
(20.54)\end{array}$ & 3.04 & 1.27 \\
\hline I don't like to talk to my friends about my purchases & $\begin{array}{c}97 \\
(10.43)\end{array}$ & $\begin{array}{c}268 \\
(28.82)\end{array}$ & $\begin{array}{c}277 \\
(29.79)\end{array}$ & $\begin{array}{c}119 \\
(12.79)\end{array}$ & $\begin{array}{c}169 \\
(18.17)\end{array}$ & 3.00 & 1.24 \\
\hline I don't like to talk to my friends about my purchases & $\begin{array}{c}107 \\
(11.50)\end{array}$ & $\begin{array}{c}241 \\
(25.92)\end{array}$ & $\begin{array}{c}263 \\
(28.28)\end{array}$ & $\begin{array}{c}127 \\
(13.66)\end{array}$ & $\begin{array}{c}192 \\
(20.65)\end{array}$ & 3.06 & 1.29 \\
\hline I often read advertisements just out of curiosity & $\begin{array}{c}104 \\
(11.18)\end{array}$ & $\begin{array}{c}415 \\
(44.63)\end{array}$ & $\begin{array}{c}283 \\
(30.43)\end{array}$ & $\begin{array}{c}76 \\
(8.17)\end{array}$ & $\begin{array}{c}52 \\
(5.60)\end{array}$ & 3.47 & 0.98 \\
\hline
\end{tabular}

Fig.1 Structural equation model of Theory of Planned Behaviour denoted by the relationship between latent variable B and BI and indicator variables A, SN, PBC.RMSEA $=0.11$

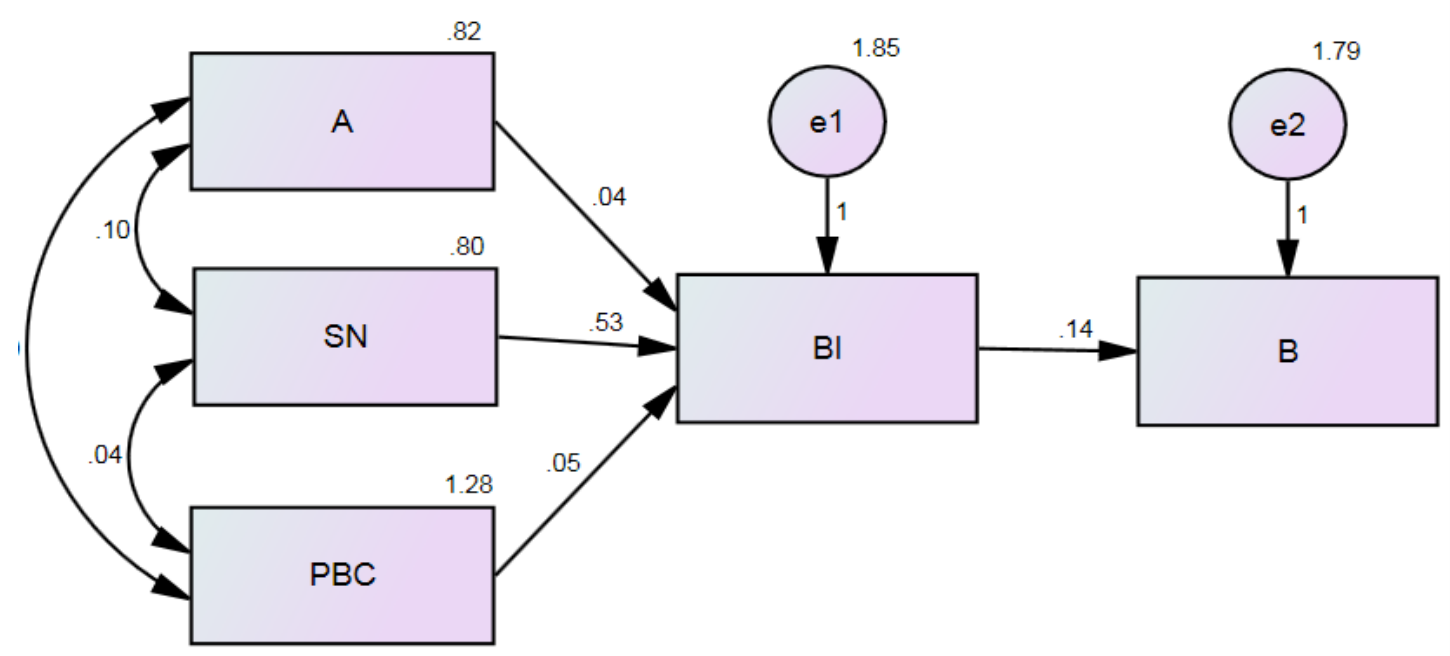

\begin{tabular}{|l|l|l|l|}
\hline & & & Estimate \\
\hline BI & $<---$ & A & .04 \\
\hline BI & $<---$ & SN &. $\mathbf{5 3}$ \\
\hline BI & $<---$ & PBC & .05 \\
\hline B & $<---$ & BI & .14 \\
\hline
\end{tabular}


Fig.2 Demographic profiling of the respondents $(n=930)$
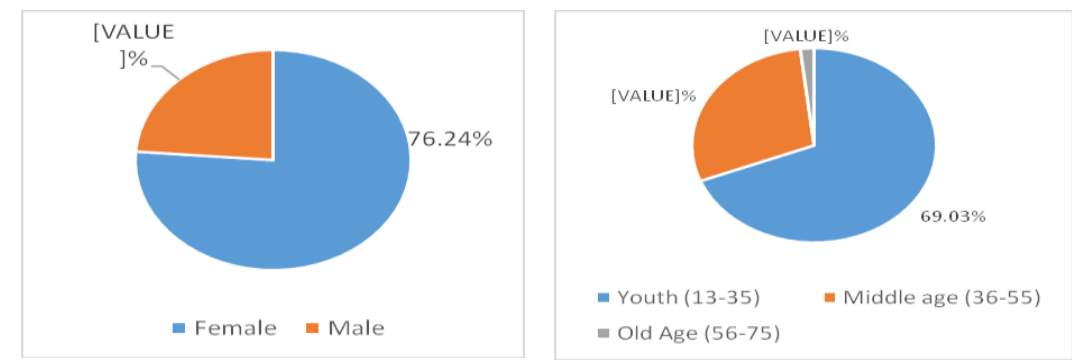

Fig.3 Scale of ranking as per the Schwartz's value best-worst survey
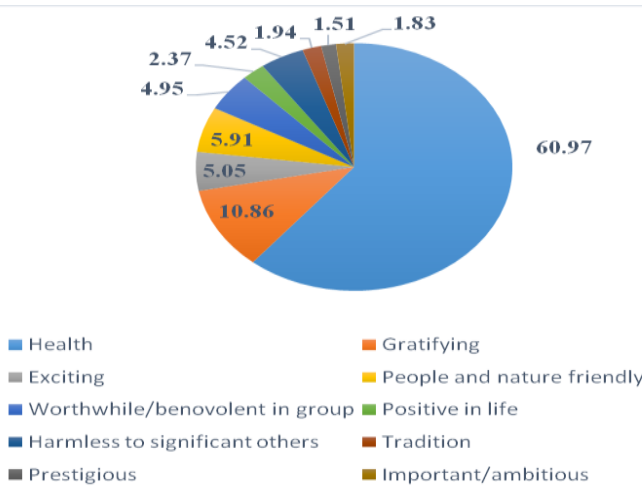

Fig.4 Consumer's knowledge towards fortified food

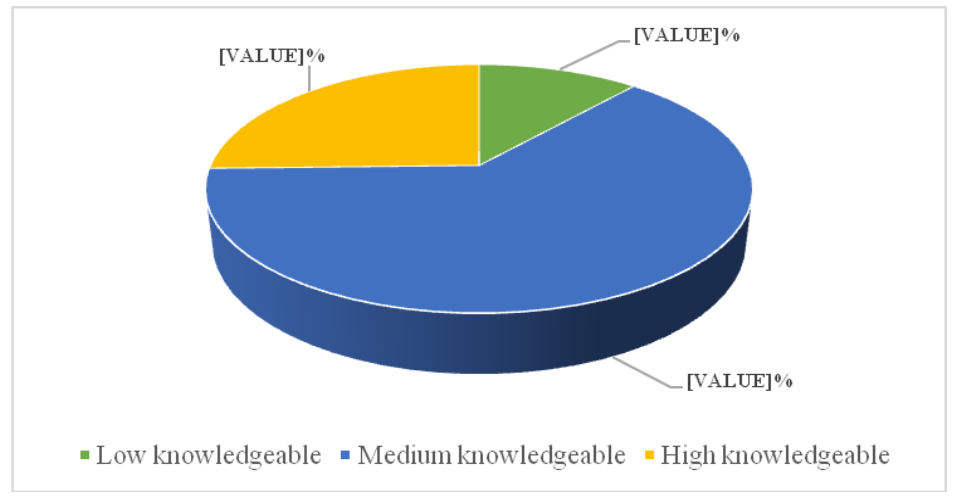

As discussed above consumer's knowledge is an important determinant that helps in forming the consumer's attitude towards purchasing fortified food. So, it was worthy to collect the information about the knowledge levels amongst the urban population about the fortified foods. The knowledge test was pre tested upon 42 individual who were not included in the final test to avoid pre testing effect. Difficulty index, discrimination index and point biserial correlation co-efficient was calculated for inclusion of items in the knowledge test. So, on the basis of above mentioned criteria four questions were screened out due to not having acceptable values. Out of total 26 knowledge items, 22 items were selected based on difficulty index ranging from 30-80, discrimination index 
exceeding 0.20 and significant bi-serial correlation co-efficient. The data for knowledge test reflected that only 27.19 percent were completely aware of the term 'fortified food' whereas, majority of the consumers $(67.74 \%)$ said that they were moderately familiar with the term while 12.21 percentage of the respondents had low level of knowledge about the same (Fig 4). A major challenge for food marketers is to improve understanding of the functional/fortified foods and communicate benefits, while satisfying regulatory requirements. In a study conducted by Brecic et al., (2012) in an eastern European country 'Croatia', the knowledge about the functional foods was mixed amongst the respondents, with only just over one quarter of them telling themselves as either very well or fully informed. It is believed that poor knowledge or conflicting information is particularly likely to discourage the consumers to go for newly launched fortified food items in the market. Further, the proper information on to the packaged food labels play a crucial role in generating knowledge to the consumers and changes their perception to buy the food products. This has been supported by a study conducted by Seo et al., (2014) on the consumption of processed food signifying that respondents with less information did not trust product labels, had little intention of buying processed foods, and wanted processed foods only if the price was reasonable. Thus, creating awareness and knowledge about fortified food products amongst the consumers' could play an important role in up gradation of fortified food marketing in our country.

Now, knowledge pertaining to the consumption of fortified food is directly related to the availability of these products. Thus, it was important to measure the perception aspects of the fortified food in context of their availability, affordability and accessibility (table 2). The same was calculated on the 7 point continuum scale ranging from extremely good to extremely bad. It was found that majority of the respondents living in Delhi answered the good availability of fortified food items in the market, but affordability of such products was one of the considerable factor hindering the frequent consumption.

After gathering information about the knowledge and perception it was of prime interest to know about such more factors that could influence the purchase behavior of the consumers. It can be seen from the table (table 3) that in general the nutritional composition of the product followed by the food safety and appropriate knowledge about the particular product were some of the prime factors that consumers take in consideration while purchasing any food item.

It was also found that price value of the product, acceptable taste and availability of fortified products were also important factors that could influence the purchase behavior of the consumers. In support, Seo et al., (2014) also concluded that price, nutritional information, and taste are some of the important motivatin gfactors that influence the consumption of foods.

Since 'affordability' and low price emerged as an important consideration for consumers as revealed in table 2 and 3 , the Willingness to pay (WTP) for fortified foods was also calculated. The concept of WTPis defined as the maximum price a given consumer accepts to pay for a product or service (Sriwaranun $e t$ al., 2014). In the current study also the WTP of the Delhi consumers towards purchasing fortified food products was calculated (table 4). Data pertaining to the same signified that majority of the respondents i.e. mean value of 4.84 (on a scale of 1-7) answered that they can only consider buying organic foods if 
they are more or less the same price as conventional food. On the other side, slightly lesser value i.e. 4.65 average of the surveyed population replied they only tend to buy fortified products when they are cheaper in price. Although, willingness to pay depends on the price value of the product, some relevant specifications could help consumers to buy and purchase such products at slightly reasonable price too.

A recent study carried out by Dolgopolova and Teuber (2018) noted an interesting and valid point saying that informing about the specific health benefits provided by a particular functional food products for instance "lowering cholesterol" significantly increases the willing to pay amongst consumers. Van Wezemael et al., (2014) concluded that specification on nutrition and health claims receives high valuations than any other claims. Further, presence of a healthy substances say vitamins or cholesterol lowering fatty acids like omega-3 fatty acid, oryzanols etc. also play important role in improving the willingness to pay by the consumers having proper knowledge of the role provided by such active substances in health. Also, labelling and information to the consumers also helps in illustrating or communicating the value of the product in term of health management. Clear and crisp information about the healthy substances present in food product also initiates a positive WTP by the consumers (Dolgopolova and Teuber, 2018).

It was clear from the table 4 that majority of the metro consumers tends to purchase fortified food products when they are more or less same in price, it would be helpful to the manufacturer if some other barriers could also be notified for not preferring such health foods. The same was calculated on a 7 continuum scale ranging from extremely important to not at all important (table 5).
Results illustrated that apart from the price value (5.98), fear of the artificial additives (5.29) and fear of any side effects (5.17) from fortified/functional food emerged as the major concern for not purchasing such products. However, failure in meeting the expectations in context of their effectiveness towards health and not acceptable sensorial attributes were some of the other factors that influences the overall acceptability of these health products.

As discussed above that proper knowledge about the fortified food items may help in further increment in the consumption of such products, when asked about the future prospects of these good number of the respondents (815) replied that yes they believe, people will buy fortified food as they will be more aware about the term (table 6). Majority of the respondents said that future generation is going to be more health conscious and hence demand for the fortified food items will increase with time, followed by the statement indicating that they consider consumption of fortified food healthier.

\section{Exploratory consumer buying behavior}

Exploratory consumer buying behavior is grounded on the basic psychological theory of optimum stimulation level and exploratory behavior and is reliable with a variety of distinctions in the consumer behavior literature between sensory and cognitive forms of stimulation seeking (Baumgartner and Steenkamp, 1996). In the current study the exploratory behavior of the consumers was measured using a 5 continuum scale ranging from strongly agree to disagree and it was found that (table 7) consumers agreed that even though certain food products are available in a number of different flavor, they tend to buy the same flavor. Which in turn indicated that sensorial attributes of food products play an important role for purchase 
behavior. Moreover, it was perceived that consumers are not ready to try something new with new flavors rather feel safe consuming the existing products or dishes. Also, the risk taking abilities in consumers was found to be less agreeing with the statement that 'If I like a brand, I rarely switch from it just to try something different'. Which might be due to the expensiveness of the fortified food or not acceptable flavor.

In conclusion the fortified food industry is progressing worldwide. Nevertheless, thriving commercialization of fortified food products will be a challenge. The foremost underlying factor is how consumers identify functional foods and what issues influence an affirmative attitude in their buying decision. It was bring into being that the theory of planned behavior can help in understand the consumers' intention in a clear manner. In this case, TPB model constructed demonstrated a quite good fitness, indicating the positive intention of the surveyed population to purchase fortified items which was found to be majorly governed by subjective norms.

Revelations from demographic details of the respondents revealed that females were more actively involved in the purchase of fortified commodities and it was realized that value of health aspect towards living their life's as well as for consuming fortified foods was on the 1st rank, which was found to be in correlation with the perspective of respondents towards health consciousness.

Moreover, it was perceived that fortified food is capturing the market rapidly, but lack of proper knowledge and high cost of the commodities are some of the significant factors creating negative impact on consumption of fortified food items. Overall, this study has
Contributed to further understanding of the fortified food consumer especially concerning health awareness, lifestyle changes and factors encouraging as well as discouraging people to purchase fortified foods. According to Frewer et al., (2003), the assumption that fortified foods with specific health advantages are likely to deliver population-wide benefits may not automatically hold. Understanding the consumer is going to be the "key" in determining whether the fortified food concept will be sustainable and will achieve the intended results.

\section{References}

Ajzen, I., The theory of planned behavior. Organizational Behavior and Human Decision Processes, 1991, 50, 179-211.

Ajzen, I., Constructing a theory of planned behavior questionnaire: Conceptual and methodological considerations, 2002, Retrieved June 1, 2005, from http://wwwunix.oit.umass.edu/\%7Eaize n/pdf/tpb.measurement.pdf $>$.

Ajzen, I., From decisions to actions: A theory of planned behavior. In: Kuhl J, Beckmann J, editors. Action-control: From cognition to behavior. Springer; New York: 1985. pp. 11-39.

Ajzen, I., Fishbein, M., Understanding attitudes and predicting social behavior. Prentice Hall; Englewood Cliffs, NJ, 1980.

Anttolainen, M., Luoto, R., Uutela, A., Boice, J.D. Jr, Blot, W.J., McLaughlin, J.K., Puska, P., Characteristics of users and nonusers of plant stanol ester margarine in Finland: an approach to study functional foods. Journal of the American Dietetic Association, 2001, 101(11), 1365-1368.

Armitage, C. J., \& Conner, M., Efficacy of the theory of planned behaviour: A meta-analytic review. British Journal of Social Psychology, 2001, 40, 471-499. 
Borgmeier, I., Westenhoefer, J., Impact of different food label formats on healthiness evaluation and food choice of consumers: a randomized-controlled study. BMC Public Health, 2009, 9, 184.

Brecic, R., Gorton, M., Barjolle, D., Understanding variations in the consumption of functional foods evidence from Croatia. British Food Journal, 2014, 116 (4), 662-675.

Childs, N.M., Poryzees, G.H., Foods that help prevent disease: consumer attitudes and public policy implications. British Food Journal, 1997, 100 (9), 419-426.

Cox, D. N., Bastiaans, K., Understanding Australian consumers' perceptions of selenium and motivations to consume selenium enriched foods. Food Quality and Preference, 2007, 18, 66-76.

Cox, D. N., Koster, A., Russell, C. G., Predicting intentions to consume functional foods and supplements to offset memory loss using an adaptation of protection motivation theory. Appetite, 2004, 43, 55-64.

Cranfield, J., Henson, S., Masakure, O., Factors affecting the extent to which consumers incorporate functional ingredients into their diets. Journal of Agricultural Economics, 201162 (2), 375-392.

Dolgopolova, I., Teuber, R., Consumers' Willingness to Pay for Health Benefits in Food Products: A Meta-Analysis. Applied Economic Perspectives and Policy, 2018, 40 (2), 333-352.

Frewer, L., Scholderer, J., Lambert, N., Consumer acceptance of functional foods: issues for the future. British Food Journal, 2003, 105(10), 714-731.

Huchting, K., Lac, A., LaBrie, J. W., An Application of the Theory of Planned Behavior to sorority alcohol consumption. Addictive Behaviors, 2008, 33(4), 538-551.
Loehlin, J.C., Latent Variable Models: An Introduction to Factor, Path and Structural Equation Analysis. Lawrence Erlbaum Assoc Inc., 2004.

Mannar, M.G., Food fortification. Encyclopedia of Food Sciences and Nutrition 2003, 2613-2621.

Markovina, J., Acicc, C., Gajdos, J., Kljusuric, J., Kovacic, D., Young consumers perception of functional foods in Croatia. British Food Journal, 2011, 113(1), 7-16.

Niva, M., Can we predict who adopts healthpromoting foods? Users of functional foods in Finland. Scandinavian Journal of Food and Nutrition, 2006, 50(1), 1324.

Onwezen, M. C., Bartels, J., Which perceived characteristics make product innovations appealing to the consumer? A study on the acceptance of fruit innovations using cross-cultural consumer segmentation. Appetite, 2011, 57(1), 50-58.

Patwardhan, B., Warude, D., Pushpangadan, P., Bhatt, N., Ayurveda and Traditional Chinese Medicine: a comparative overview. Evid Based Complement Alternat Med, 2005, 2(4), 465-473

Poulsen, J.B., Danish consumers' attitudes towards functional foods. Working paper no 62, MAPP, Århus, Denmark, 1999. Report of Assocham-EY, 2017.

Salleh, M. M., Ali, S. M., Harun, E. H., Jalil, M. A., Shaharudin, M. R., Consumer's perception and purchase intentions towards organic food products: Exploring attitude among academician. Canadian Social Science 2010, 6(6), 119-129.

Schwartz, S. H., Universals in the Content and Structure of Values: Theoretical Advances and Empirical tests in 20 countries. Advances in Experimental Social Psychology, 1992, 25, 1-65.

Schwartz, S. H., Are there Universal Aspects 
in the Structure and Content of Human Values? Journal of Social Issues, 1994, 50(4), 19-45.

Seo, S., Kim, O.Y., Shim, S., Using the theory of planned behavior to determine factors influencing processed foods consumption behavior. Nutrition Research and Practice, 2014, 8(3), 327335.

Siro, I., Kápolna, E., Kápolna, B., Lugasi, A., Functional Food. Product Development, Marketing and Consumer Acceptance-a Review. Appetite, 2008, 51 (3), 45667.

Sriwaranun, Y., Gan, C., Lee, M., Cohen, D.A., Consumers' willingness to pay for organic products in Thailand. International Journal of Social Economics, 2015, 42 (5), 480-510.

Steptoe, A., Pollard, T.M., Wardle, J., Development of a measure of the motives underlying the selection of food: the food choice questionnaire. Appetite, 1995, 25, 267-284.
Tapsell, L. C., Functional foods: An Australian perspective. Nutrition and Dietetics, 2008, 65(3), 23-26.

Urala, N., Lähteenmäki, L.,Strength of healthrelated claims and their perceived advantage. International Journal of Food Science and Technology, 2003, $38,218-826$.

Urala, N., Lähteenmäki, L., Attitudes behind consumers' willingness to use functional foods. Food Quality and Preference, 2004, 15, 793-803.

Van Wezemael L., Caputo V., Nayga R. M., Chryssochoidis G., Verbeke W., European Consumer Preferences for Beef with Nutrition and Health Claims: A Multi-Country Investigation Using Discrete Choice Experiments. Food Policy, 2014, 44, 167-76.

Verbeke, W., Functional foods: consumer willingness to compromise on taste for health? Food Quality and Preference, 2006, 17 (1), 126-131.

\section{How to cite this article:}

Satyapriya, Jitender Kumar Chauhan and Sitaram Bishnoi. 2021. Willingness to Pay and Consumer's Behavioral Intentions towards Fortified Foods in Metropole. Int.J.Curr.Microbiol.App.Sci. 10(01): 1876-1871. doi: https://doi.org/10.20546/ijcmas.2021.1001.220 